\title{
Vector Supersymmetry of Three Dimensional Cohomological Field Theories
}

\author{
C. Linhares de Jesus, C.A. Sasaki, S.P. Sorella \\ UERJ, Universidade do Estado do Rio de Janeiro \\ Departamento de Física Teórica \\ Instituto de Física, UERJ \\ Rua São Francisco Xavier, 524 \\ 20550-013, Maracanã, Rio de Janeiro, Brazil \\ Received September 10, 1997
}

\begin{abstract}
The existence of the vector supersymmetry for three dimensional cohomological field theories is discussed. The example of the three dimensional topological model of Baulieu-Grossman
\end{abstract} [1] is considered in detail.

\section{Introduction}

Almost all known topological field theories (TFT) [2], of both Schwartz and cohomological type, turn out to be characterized by an additional invariance whose generators possess a Lorentz index and give rise, together with the BRST generator, to a supersymmetric algebra of the Wess-Zumino type.

This additional invariance, usually called vector supersymmetry, has been first detected in the case of the three dimensional Chern-Simons theory $[2,3]$ and later on has been extended to others TFT as, for instance, the BF models in different space-time dimensions [4], the bosonic string and its supersymmetric version [5], the $W_{3}$-gravity [6] and the Witten's topological euclidean Yang-Mills theory in four dimensions [7].

The vector supersymmetry has been proven to be an important tool in the characterization of the relevant BRST cohomology classes as well as in the understanding of the ultraviolet finiteness properties of TFT $[2,4]$. It is also worthwhile to mention here that, recently, the existence of the vector susy has been related to the twisted version of extended supersymmetries, strengthening thus the deep relationship between TFT and supersymmetric models [7].

The aim of this work is to investigate the presence of the vector susy for the three dimensional TFT of the cohomological type. As a prototype of this class of TFT we shall consider in detail the model proposed by $\mathrm{L}$.
Baulieu and B. Grossman [1] in order to describe topological invariants related to three dimensional magnetic monopoles.

The work is organized as follows. In Sect.II we present the model and we discuss the existence of the vector susy. Sect.III is devoted to the derivation of a generalized Slavnov-Taylor identity which collects together both the BRST and the vector susy transformations. Finally, in Sect.IV we discuss the cohomology of the generalized Slavnov-Taylor operator and we present the renormalization of the model.

\section{The action and its supersymmetric structure}

The model proposed by L. Baulieu and B. Grossman is a three dimensional cohomological theory of the Yang-Mills type. Following [1], the field content of the model is given by the set $\left(A_{\mu}, \varphi, \psi_{\mu}, \chi_{\mu}, \xi, \eta, \phi, \bar{\phi}\right)$, respectively a gauge connection $A_{\mu}$, a real scalar $\varphi$, two anticommuting vector fields $\left(\psi_{\mu}, \chi_{\mu}\right)$, two anticommuting scalar fields $(\xi, \eta)$ as well as a pair of complex commuting scalars $(\phi, \bar{\phi})$. Moreover, introducing the usual Faddeev-Popov ghosts $(c, \bar{c})$ and a pair of Lagrange multipliers $\left(B, b_{\mu}\right)$, for the BRST transformations we have

$$
\begin{aligned}
s A_{\mu} & =D_{\mu} c+\psi_{\mu}, \\
s \psi_{\mu} & =-D_{\mu} \phi-g\left\{c, \psi_{\mu}\right\},
\end{aligned}
$$




$$
\begin{aligned}
s \xi & =g[\phi, \varphi]-g\{c, \xi\}, \\
s \varphi & =\xi-g[c, \varphi], \\
s \phi & =-g[c, \phi], \\
s c & =\phi-g c^{2}, \\
s \bar{\phi} & =\eta, \quad s \eta=0, \\
s \bar{c} & =B, \quad s B=0, \\
s \chi_{\mu} & =b_{\mu}, \quad s b_{\mu}=0, s^{2}=0,
\end{aligned}
$$

where $D_{\mu}$ is the covariant derivative, defined as

$$
D_{\mu} \cdot=\partial_{\mu} \cdot+g\left[A_{\mu}, \cdot\right],
$$

$g$ being the gauge coupling constant with mass dimension $1 / 2$. Notice that the BRST variation of the gauge connection $A_{\mu}$ is characterized, besides the usual pure gauge term $\left(D_{\mu} c\right)$, by an additional shift transformations $\left(\psi_{\mu}\right)$ which is typical of the TFT of the cohomological type $[2,7]$. The quantum numbers of all the fields are assigned as follows:

\begin{tabular}{|l|l|l|l|l|l|l|l|l|l|l|l|l|}
\hline 0 & $A_{\mu}$ & $\varphi$ & $\psi_{\mu}$ & $\chi_{\mu}$ & $\xi$ & $\eta$ & $\phi$ & $\bar{\phi}$ & $c$ & $\bar{c}$ & $B$ & $b_{\mu}$ \\
\hline dim. & $1 / 2$ & $1 / 2$ & 1 & 1 & 1 & 1 & $1 / 2$ & $1 / 2$ & 0 & 1 & $3 / 2$ & $3 / 2$ \\
\hline gh. numb. & 0 & 0 & 1 & -1 & 1 & -1 & 2 & -2 & 1 & -1 & 0 & 0 \\
\hline
\end{tabular}

As one can expect due the cohomological nature of the model, the classical invariant gauge fixed action can be expressed as a pure BRST variation, namely

$$
\begin{array}{r}
S_{i n v}=s \int d^{3} x \operatorname{tr}\left(\chi^{\mu} D_{\mu} \varphi+\varepsilon^{\mu \nu \rho} \chi_{\mu} F_{\nu \rho}+\frac{1}{2} \chi^{\mu} b_{\mu}\right. \\
\left.+\bar{c}\left(\partial A+\frac{B}{2}\right)+\bar{\phi}\left(D_{\mu} \psi^{\mu}+g[\xi, \varphi]\right)\right),
\end{array}
$$

where a Feynman like gauge-fixing condition has been adopted [1]. Expression (2.4) is easily worked out and yields

$$
\begin{aligned}
S_{i n v}= & t r \int d^{3} x\left(b^{\mu} D_{\mu} \varphi-\chi^{\mu} D_{\mu} \xi+g \chi^{\mu}\left[c, D_{\mu} \varphi\right]-g \chi^{\mu}\left[\psi_{\mu}, \varphi\right]\right. \\
& +\varepsilon^{\mu \nu \rho} b_{\mu} F_{\nu \rho}+g \varepsilon^{\mu \nu \rho} \chi_{\mu}\left[c, F_{\nu \rho}\right]-2 \varepsilon^{\mu \nu \rho} \chi_{\mu}\left[c, F_{\nu \rho}\right]+\frac{1}{2} b^{\mu} b_{\mu} \\
& -2 \varepsilon^{\mu \nu \rho} \chi_{\mu} D_{\nu} \psi_{\rho}+B\left(\partial A+\frac{B}{2}\right)-\bar{c} \partial^{\mu} D_{\mu} c-\bar{c} \partial \psi \\
& +\eta\left(D_{\mu} \psi^{\mu}+g[\xi, \varphi]\right)-\bar{\phi} D^{\mu} D_{\mu} \phi-g \bar{\phi}\left\{c, D^{\mu} \psi_{\mu}\right\} \\
& \left.+g \bar{\phi}\left\{\psi^{\mu}, \psi_{\mu}\right\}-g^{2} \bar{\phi}\{c,[\xi, \varphi]\}-g \bar{\phi}\{\xi, \xi\}+g^{2} \bar{\phi}[[\phi, \varphi], \varphi]\right) .
\end{aligned}
$$

Let us also notice that the term

$$
\operatorname{tr} \int d^{3} x\left(b^{\mu} D_{\mu} \varphi+\varepsilon^{\mu \nu v} b_{\mu} F_{\nu v}+\frac{b^{\mu} b_{\mu}}{2}\right),
$$

is equivalent to a standard Yang-Mills action

$$
-\operatorname{tr} \int d^{3} x\left(\frac{1}{2} D^{\mu} \varphi D_{\mu} \varphi+F_{\mu \nu} F^{\mu \nu}\right)
$$

upon elimination of the Lagrange multiplier $b^{\mu}$. In order to detect the supersymmetric structure of the classical action (2.4), (2.5) we introduce the following vector type operator $\delta_{\mu}$ with negative ghost number, defined as 


$$
\begin{aligned}
\delta_{\mu} c & =A_{\mu}, \\
\delta_{\mu} \phi & =-\psi_{\mu}, \\
\delta_{\mu} A_{\nu} & =0, \\
\delta_{\mu} \psi_{\nu} & =F_{\mu \nu}, \\
\delta_{\mu} \varphi & =0, \\
\delta_{\mu} \xi & =D_{\mu} \varphi, \\
\delta_{\mu} \eta & =\partial_{\mu} \bar{\phi}, \\
\delta_{\mu} \bar{\phi} & =0, \\
\delta_{\mu} B & =\partial_{\mu} \bar{c}, \\
\delta_{\mu} \bar{c} & =0, \\
\delta_{\mu} b_{\nu} & =\partial_{\mu} \chi_{\nu}, \\
\delta_{\mu} \chi_{\nu} & =0 .
\end{aligned}
$$

The operator $\delta_{\mu}$ acts nonlinearly on the fields and gives rise, together with the BRST operator $s$, to the following anticommutation relations

$$
\begin{aligned}
\left\{s, \delta_{\mu}\right\} & =\partial_{\mu}, \\
\left\{\delta_{\mu}, \delta_{\nu}\right\} & =0 .
\end{aligned}
$$

We see therefore that the algebra between $s$ and $\delta_{\mu}$ closes on the space-time translations, allowing thus for a supersymmetric interpretation.

Having identified the supersymmetric structure we were looking for, let us now check if the $\delta_{\mu}$-transformations of eq. (2.8) leave the action (2.4) invariant. After a little algebra we easily obtain

$$
\delta_{\mu} S_{i n v}=s O_{\mu}
$$

with

$$
O_{\mu}=-\operatorname{tr} \int d^{3} x\left(\bar{\phi}\left(D^{\nu} F_{\mu \nu}+g\left[D_{\mu} \varphi, \varphi\right]\right)-\frac{1}{2} \chi^{v} \partial_{\mu} \chi_{\nu}-\frac{1}{2} \bar{c} \partial_{\mu} \bar{c}\right)
$$

showing thus that the vector transformations (2.8) do not actually correspond to an exact symmetry of the action $S_{i n v}$. However, since the breaking term appearing on the right hand side of eq. $(2.10)$ is a pure BRST variation and since

$$
\delta_{\mu} O_{\nu}=0
$$

we can use a very useful trick in order to turn $\delta_{\mu}$ an exact symmetry of a suitable modified action. Following indeed a well known procedure $[4,7]$, we first collect all the generators entering the algebra (2.9) into a unique generalized nilpotent operator $\mathcal{Q}$.

Introducing then two constant ghosts $\left(\varepsilon^{\mu}, \vartheta^{\mu}\right)$, associated respectively to the vector generators $\delta_{\mu}$ and to the space-time translations $\partial_{\mu}$, 


\begin{tabular}{|c|c|c|}
\multicolumn{3}{c}{ Table2 } \\
\hline dim. & $\varepsilon^{\mu}$ & $\vartheta^{\mu}$ \\
\hline gh. numb. & 0 & $-1 / 2$ \\
\hline
\end{tabular}

it is easily verified that the generalized operator $\mathcal{Q}$ defined as

$$
\mathcal{Q}=s+\varepsilon^{\mu} \delta_{\mu}+\vartheta^{\mu} \partial_{\mu}-\varepsilon^{\mu} \frac{\partial}{\partial \vartheta^{\mu}},
$$

turns out to be nilpotent,

$$
\mathcal{Q}^{2}=0
$$

From eq. (2.14) it is apparent that the operator $\mathcal{Q}$ collects together all the generators entering the relations (2.9). Acting now with $\mathcal{Q}$ on the classical invariant action $S_{\text {inv }}$, we get

$$
\mathcal{Q} S_{i n \nu}=s\left(\varepsilon^{\nu} O_{\nu}\right) \text {. }
$$

Recalling now that the breaking term $O_{\nu}$ is left invariant by $\delta_{\mu}$, (i.e. $\delta_{\mu} O_{\nu}=0$ ), we can rewrite the right-hand side of eq. (2.16) as

$$
\mathcal{Q} S_{i n \nu}=\left(s+\varepsilon^{\mu} \delta_{\mu}+\vartheta^{\mu} \partial_{\mu}-\varepsilon^{\mu} \frac{\partial}{\partial \vartheta^{\mu}}\right) \varepsilon^{\nu} O_{\nu}=\mathcal{Q}\left(\varepsilon^{\nu} O_{\nu}\right)
$$

i.e.

$$
\mathcal{Q}\left(S_{\text {inv }}-\varepsilon^{\nu} O_{\nu}\right)=0
$$

which means that the modified action

$$
\widetilde{S}_{i n v}=S_{i n v}-\varepsilon^{\nu} O_{\nu},
$$

is left invariant by the generalized operator $\mathcal{Q}$. In other words, the properties (2.10) and (2.12) allow to suitably modify the initial action (2.4) so that the generalized operator $\mathcal{Q}$ becomes an exact symmetry, while collecting together both operators $\left(s, \delta_{\mu}\right)$. We are now ready to built up the Slavnov-Taylor identity and to discuss the quantum properties of the model. This will be the task of the next Section.

\section{The Slavnov-Taylor identity}

In order to obtain the Slavnov-Taylor identity we introduce, following a standard procedure [8], a set of antifields $\left(A_{\mu}^{*}, \psi_{\mu}^{*}, \xi^{*}, \varphi^{*}, \phi^{*}, c^{*}\right)$ coupled to the nonlinear $\mathcal{Q}$-transformations of the fields $\left(A_{\mu}, \psi_{\mu}, \xi, \varphi, \phi, c\right)$,

$$
S_{e x t}=\operatorname{tr} \int d^{3} x\left(A^{* \mu} \mathcal{Q} A_{\mu}+\psi^{* \mu} \mathcal{Q} \psi_{\mu}+\xi^{*} \mathcal{Q} \xi+\varphi^{*} \mathcal{Q} \varphi+\phi^{*} \mathcal{Q} \phi+c^{*} \mathcal{Q} c\right)
$$

Defining then the complete action $\Sigma$ as

$$
\Sigma=\widetilde{S}_{i n v}+S_{e x t}
$$

for the classical Slavnov-Taylor identity we get

$$
\mathcal{S}(\Sigma)=0
$$

with 


$$
\begin{aligned}
\mathcal{S}(\Sigma)=\operatorname{tr} & \int d^{3} x\left(\frac{\delta \Sigma}{\delta A^{* \mu}} \frac{\delta \Sigma}{\delta A_{\mu}}+\frac{\delta \Sigma}{\delta \psi^{* \mu}} \frac{\delta \Sigma}{\delta \psi_{\mu}}+\frac{\delta \Sigma}{\delta \xi^{*}} \frac{\delta \Sigma}{\delta \xi}+\frac{\delta \Sigma}{\delta \varphi^{*}} \frac{\delta \Sigma}{\delta \varphi}+\frac{\delta \Sigma}{\delta \phi^{*}} \frac{\delta \Sigma}{\delta \phi}\right. \\
& +\frac{\delta \Sigma}{\delta c^{*}} \frac{\delta \Sigma}{\delta c}+\left(\eta+\vartheta^{\nu} \partial_{\nu} \bar{\phi}\right) \frac{\delta \Sigma}{\delta \bar{\phi}}+\left(\varepsilon^{\nu} \partial_{\nu} \bar{\phi}+\vartheta^{\nu} \partial_{\nu} \eta\right) \frac{\delta \Sigma}{\delta \eta} \\
& +\left(B+\vartheta^{\nu} \partial_{\nu} \bar{c}\right) \frac{\delta \Sigma}{\delta \bar{c}}+\left(\varepsilon^{\nu} \partial_{\nu} \bar{c}+\vartheta^{\nu} \partial_{\nu} B\right) \frac{\delta \Sigma}{\delta B}+\left(b_{\mu}+\vartheta^{\nu} \partial_{\nu} \chi_{\mu}\right) \frac{\delta \Sigma}{\delta \chi_{\mu}} \\
& \left.+\left(\varepsilon^{\nu} \partial_{\mu} \chi_{\mu}+\vartheta^{\nu} \partial_{\nu} b_{\mu}\right) \frac{\delta \Sigma}{\delta b_{\mu}}\right)-\varepsilon^{\mu} \frac{\partial \Sigma}{\partial \vartheta^{\mu}}
\end{aligned}
$$

Furthermore, introducing the so called linearized operator $\mathcal{B}_{\Sigma}[8]$ defined as

$$
\begin{aligned}
\mathcal{B}_{\Sigma}=\operatorname{tr} & \int d^{3} x\left(\frac{\delta \Sigma}{\delta A^{* \mu}} \frac{\delta}{\delta A_{\mu}}+\frac{\delta \Sigma}{\delta A_{\mu}} \frac{\delta}{\delta A^{* \mu}}+\frac{\delta \Sigma}{\delta \psi^{* \mu}} \frac{\delta}{\delta \psi_{\mu}}+\frac{\delta \Sigma}{\delta \psi_{\mu}} \frac{\delta}{\delta \psi^{* \mu}}+\frac{\delta \Sigma}{\delta \xi^{*}} \frac{\delta}{\delta \xi}\right. \\
& +\frac{\delta \Sigma}{\delta \xi} \frac{\delta}{\delta \xi^{*}}+\frac{\delta \Sigma}{\delta \varphi^{*}} \frac{\delta}{\delta \varphi}+\frac{\delta \Sigma}{\delta \varphi} \frac{\delta}{\delta \varphi^{*}}+\frac{\delta \Sigma}{\delta \phi^{*}} \frac{\delta}{\delta \phi}+\frac{\delta \Sigma}{\delta \phi} \frac{\delta}{\delta \phi^{*}} \\
& +\frac{\delta \Sigma}{\delta c^{*}} \frac{\delta}{\delta c}+\frac{\delta \Sigma}{\delta c} \frac{\delta}{\delta c^{*}}+\left(\eta+\vartheta^{\nu} \partial_{\nu} \bar{\phi}\right) \frac{\delta}{\delta \bar{\phi}}+\left(\varepsilon^{\nu} \partial_{\nu} \bar{\phi}+\vartheta^{\nu} \partial_{\nu} \eta\right) \frac{\delta}{\delta \eta} \\
& +\left(B+\vartheta^{\nu} \partial_{\nu} \bar{c}\right) \frac{\delta}{\delta \bar{c}}+\left(\varepsilon^{\nu} \partial_{\nu} \bar{c}+\vartheta^{\nu} \partial_{\nu} B\right) \frac{\delta}{\delta B}+\left(b_{\mu}+\vartheta^{\nu} \partial_{\nu} \chi_{\mu}\right) \frac{\delta}{\delta \chi_{\mu}} \\
& \left.+\left(\varepsilon^{\nu} \partial_{\mu} \chi_{\mu}+\vartheta^{\nu} \partial_{\nu} b_{\mu}\right) \frac{\delta}{\delta b_{\mu}}\right)-\varepsilon^{\mu} \frac{\partial}{\partial \vartheta^{\mu}}
\end{aligned}
$$

one has

$$
\mathcal{B}_{\Sigma} \mathcal{B}_{\Sigma}=0
$$

The relevance of the nilpotent operator $\mathcal{B}_{\Sigma}$ is due to the fact the possible invariant counterterms and anomalies which may affect the Slavnov-Taylor at the quantum level can be identified as cohomology classes of the operator $\mathrm{B}_{\Sigma}$ with ghost-number 0 and 1 , respectively [8]. Let us turn therefore to the study of the cohomology of $\mathcal{B}_{\Sigma}$. To this purpose we introduce the filtering operator [8]

$$
\mathcal{N}=\varepsilon^{\mu} \frac{\partial}{\partial \varepsilon^{\mu}}+\vartheta^{\mu} \frac{\partial}{\partial \vartheta^{\mu}}
$$

which counts the number of global constant ghosts. Accordingly, the linearized operator $\mathcal{B}_{\Sigma}$ decomposes as

$$
\mathcal{B}_{\Sigma}=b+\varepsilon^{\mu} \mathcal{W}_{\mu}+\vartheta^{\mu} \mathcal{P}_{\mu}
$$

$\mathcal{P}_{\mu}$ being the Ward operator corresponding to the space-time translations. From the nilpotency condition (3.24) the operators $b, \mathcal{W}_{\mu}, \mathcal{P}_{\mu}$ are seen to obey the following relations

$$
\begin{gathered}
b^{2}=0, \\
\left\{b, \mathcal{W}_{\mu}\right\}=\mathcal{P}_{\mu}, \\
\left\{\mathcal{W}_{\mu}, \mathcal{W}_{\nu}\right\}=0, \quad\left[\mathcal{P}_{\mu} \mathcal{P}_{\nu}\right]=0 .
\end{gathered}
$$


Observe that, as a consequence of the algebraic relations $(2.9)$, the anticommutator between the operator $b$ and $\mathcal{W}_{\mu}$ closes on the space-time translations. Let us also give, for further use, the explicit expression of the two operators $b, \mathcal{W}_{\mu}$, namely

$$
\begin{array}{rlrl}
b \lambda & =s \lambda ; & & \lambda=\left(A_{\mu}, \psi_{\mu}, \xi, \varphi, \phi, \bar{\phi}, c, \eta, \bar{c}, B, \chi_{\mu}, b_{\mu}\right), \\
b \vartheta_{\mu}=-\varepsilon_{\mu}, & b \varepsilon_{\mu}=0,
\end{array}
$$

and

$$
\begin{aligned}
b A_{\mu}^{*}= & g[\varphi, b]+g\left\{\xi, \chi_{\mu}\right\}+g^{2}\left[\varphi,\left\{\chi_{\mu}, c\right\}\right]+2 \varepsilon_{\mu \nu \rho} D^{\nu}\left(b^{\rho}+g\left\{\chi^{\rho}, c\right\}\right) \\
& +2 g \varepsilon_{\mu \nu \rho}\left\{\psi^{\nu} \chi^{\rho}\right\}-\partial_{\mu} B-g\left\{c, \partial_{\mu} \bar{c}\right\}-g\left\{\psi_{\mu}, \eta\right\}+g\left[\bar{\phi}, D_{\mu} \phi\right] \\
& -g\left[D_{\mu} \bar{\phi} \phi\right]+g^{2}\left\{\psi_{\mu},[\bar{\phi}, c]\right\}-g\left[\phi, \psi_{\mu}^{*}\right]-g\left\{c, A_{\mu}^{*}\right\}, \\
b \psi_{\mu}^{*}= & g\left[\varphi, \chi_{\mu}\right]+2 \varepsilon_{\mu \nu \rho} D^{\nu} \chi^{\rho}-\partial_{\mu} \bar{c}+D_{\mu} \eta-g\left[D_{\mu} \bar{\phi}, c\right]-g\left[D_{\mu} c, \bar{\phi}\right] \\
& +2 g\left[\psi_{\mu}, \phi\right]-A_{\mu}^{*}+g\left[\psi_{\mu}^{*}, c\right], \\
b \xi^{*}= & -D^{\mu} \chi_{\mu}-g[\varphi, \eta]+g^{2}[\varphi,[\bar{\phi}, c]]-2 g[\xi, \phi]-g\left\{c, \xi^{*}\right\}-\varphi^{*}, \\
b \varphi^{*}= & -D^{\mu} b_{\mu}-g\left\{D^{\mu} \chi_{\mu}, c\right\}-g\left\{\chi^{\mu}, D_{\mu} c\right\}+g\{\xi, \eta\}+g^{2}\{\xi,[c, \bar{\phi}]\} \\
& -2 g^{2}[\phi,[\varphi, \bar{\phi}]]-g\left\{c, \varphi^{*}\right\}-g\left\{\chi^{\mu}, \psi_{\mu}\right\}-g\left[\phi, \xi^{*}\right], \\
b \phi^{*}= & -D^{2} \bar{\phi}+g^{2}[\varphi,[\varphi, \bar{\phi}]]+D^{\mu} \psi_{\mu}^{*}+c^{*}-g\left\{c, \phi^{*}\right\}+g\left[\varphi, \xi^{*}\right], \\
b c^{*}= & -g\left[D^{\mu} \varphi, \chi_{\mu}\right]-g \varepsilon^{\mu \nu \rho}\left[F_{\nu \rho}, \chi_{\mu}\right]-g\left[D^{\mu} \psi_{\mu}, \bar{\phi}\right] \\
& -g^{2}[[\xi, \varphi], \bar{\phi}]+D^{\mu} A_{\mu}^{*}+D^{\mu} \partial_{\mu} \bar{c}+g\left[c, c^{*}\right]+g\left[\psi^{\mu}, \psi_{\mu}\right] \\
& -g\left[\xi, \xi^{*}\right]+g\left[\varphi, \varphi^{*}\right]+g\left[\phi, \phi^{*}\right],
\end{aligned}
$$

and

$$
\begin{aligned}
\mathcal{W}_{\mu} \lambda= & \delta_{\mu} \lambda ; \quad \lambda=\left(A_{\mu}, \psi_{\mu}, \xi, \varphi, \phi, \bar{\phi}, c, \eta, \overline{c, B}, \chi_{\mu}, b_{\mu}\right) \\
\mathcal{W}_{\mu} \varepsilon_{\nu}= & 0, \quad \mathcal{W}_{\mu} \vartheta_{\nu}=0 \\
\mathcal{W}_{\mu} A_{\nu}^{*}= & g\left[\bar{\phi}, F_{\mu \nu}\right]-D_{\mu} D_{\nu} \bar{\phi}+\delta_{\mu \nu} D^{2} \bar{\phi}+g \delta_{\mu \nu}[\varphi,[\varphi, \bar{\phi}]] \\
& -D_{\mu} \psi_{\nu}^{*}+\delta_{\mu \nu} D^{\sigma} \psi_{\sigma}^{*}+g \delta_{\mu \nu}\left[\varphi, \xi^{*}\right]+\delta_{\mu \nu} c^{*}, \\
\mathcal{W}_{\mu} \psi_{\nu}^{*}= & \delta_{\mu \nu} \phi^{*}, \\
\mathcal{W}_{\mu} \xi^{*}= & 0 \\
\mathcal{W}_{\mu} \varphi^{*}= & -2 g\left[D_{\mu} \varphi, \bar{\phi}\right]-D_{\mu} \xi^{*}, \\
\mathcal{W}_{\mu} \phi^{*}= & 0 \\
\mathcal{W}_{\mu} c^{*}= & 0 .
\end{aligned}
$$

\section{BRST Cohomology and Renormalizability}

We face now the problem of characterizing the cohomology of the linearized operator $\mathcal{B}_{\Sigma}$. To this purpose let us first compute the cohomology of the operator $b$, whose relevance relies on a very general theorem on the
BRST cohomology stating that the cohomology of the complete operator $\mathcal{B}_{\Sigma}$ is isomorphic to a subspace of the cohomology of the operator $b[8]$. To compute the cohomology of $b$ we introduce a second filtering operator $\mathcal{N}_{\lambda}$ 


$$
\begin{aligned}
\mathcal{N}_{\lambda} & =\sum_{\lambda} \int d^{3} x\left(\lambda \frac{\delta}{\delta \lambda}+\lambda^{*} \frac{\delta}{\delta \lambda^{*}}\right) \\
\lambda & =\left(A_{\mu}, \psi_{\mu}, \xi, \varphi, \phi, \bar{\phi}, c, \eta, \bar{c} B, \chi_{\mu}, b_{\mu}\right) \\
\lambda^{*} & =\left(A_{\mu}^{*}, \psi_{\mu}^{*}, \xi^{*}, \varphi^{*}, \phi^{*}, c^{*}\right)
\end{aligned}
$$

according to the which the operator $b$ decomposes as

$$
b=b_{0}+b_{1}+b_{2},
$$

with

$$
\begin{array}{rlrl}
b_{0} \varphi & =\xi, & & b_{0} \xi=0, \\
b_{0} c & =\phi, & & b_{0} \phi=0, \\
b_{0} \bar{\phi} & =\eta, & & b_{0} \eta=0, \\
b_{0} \bar{c} & =B, & & b_{0} B=0, \\
b_{0} \chi_{\mu} & =b_{\mu}, & & b_{0} b_{\mu}=0, \\
b_{0} A_{\mu} & =\partial_{\mu} c+\psi_{\mu}, & \\
b_{0} \psi_{\mu} & =-\partial_{\mu} \phi, & \\
b_{0} A_{\mu}^{*} & =2 \varepsilon_{\mu \nu \rho} \partial^{\nu} b^{\rho}-\partial_{\mu} B \\
b_{0} \psi_{\mu}^{*} & =-A_{\mu}^{*}+2 \varepsilon_{\mu \nu \rho} \partial^{\nu} \chi^{\rho}-\partial_{\mu} \bar{c}+\partial_{\mu} \eta \\
b_{0} \xi^{*} & =-\partial_{\mu} \chi^{\mu}-\varphi^{*}, \\
b_{0} \varphi^{*} & =-\partial_{\mu} b^{\mu}, & \\
b_{0} \phi^{*} & =-\partial^{2} \bar{\phi}+\partial_{\mu} \psi^{* \mu}+c^{*} \\
b_{0} c^{*} & =\partial^{\mu} A_{\mu}^{*}+\partial^{2} \bar{c} .
\end{array}
$$

Making then the following linear changes of variables

$$
\begin{aligned}
& \psi_{\mu} \longmapsto \widehat{\psi}_{\mu}=\psi_{\mu}+\partial_{\mu} c \\
& A_{\mu}^{*} \longmapsto \hat{A}_{\mu}^{*}=-\left(A_{\mu}^{*}-2 \varepsilon_{\mu \nu \rho} \partial^{\nu} \chi^{\rho}+\partial_{\mu} \bar{c}\right), \\
& \psi_{\mu}^{*} \longmapsto \hat{\psi}_{\mu}^{*}=\psi_{\mu}^{*}-\partial_{\mu} \bar{\phi} \\
& \varphi^{*} \longmapsto \hat{\varphi}^{*}=-\left(\varphi^{*}+\partial^{\mu} \chi_{\mu}\right) \\
& c^{*} \longmapsto \widehat{c}^{*}=c^{*}+\partial^{\mu} \psi_{\mu}^{*}-\partial^{2} \bar{\phi}
\end{aligned}
$$

it is easily verified that $b_{0}$-transformations of all the fields, ghosts and antifields can be cast in the form of BRST doublets $(u, v)[8]$; i.e.

$$
b_{0} u=v, \quad b_{0} v=0
$$

$$
\begin{aligned}
u & =\left(\varphi, c, \bar{\phi}, \bar{c}, \chi, A, \hat{\psi}^{*}, \xi^{*}, \phi^{*}\right), \\
v & =\left(\xi, \phi, \eta, B, b, \widehat{\psi}, \widehat{A}^{*}, \hat{\varphi}^{*}, \widehat{c}^{*}\right)
\end{aligned}
$$

It is known that the doublets do not contribute to the BRST cohomology [8], meaning that the cohomology of $b_{0}$ is empty. Therefore the cohomology of the operator $b$ in eqs. (3.30), (3.31) vanishes as well, implying in turn that the cohomology of the complete operator $\mathcal{B}_{\Sigma}$ of eq. (3.23) is empty too. From this result we infer that the Slavnov-Taylor (3.21) identity is anomaly free and that the possible invariant counterterms which can be freely added to the classical action $\Sigma$ of eq. (3.20) can be always written as pure $\mathcal{B}_{\Sigma}$-variations. As expected, this means that the quantum corrections will preserve the topological character of the Baulieu-Grossman model.

\section{V.Conclusion}

The existence of the vector supersymmmetry for the three dimensional cohomological field theory proposed by Baulieu-Grossman [1] has been investigated. We have been able to show that the model is anomaly free and that the quantum corrections preserve its topological character. Let us also notice that the model of Baulieu-Grossman has the same field content of the $N=4$ supersymmetric twisted three dimensional YangMills theory [9]. This property suggests a deeper relationship between $N=4$ three-dimensional Yang-Mills and topological theories of the cohomological type. As in the case of the Witten's four dimensional topological Yang-Mills [7], this could provide a conventional field theory framework for the so called equivariant cohomology proposed by [10] in order to identify the topological observables of the theory. This aspect as wall as the relationship of the Baulieu-Grossman model with the Witten's topological Yang-Mills through dimensional reduction are under investigation.

\section{Acknowledgements}

The Conselho Nacional de Pesquisa e Desenvolvimento, $\mathrm{CNP} q$ Brazil and the Faperj, Fundação de Amparo à Pesquisa do Estado do Rio de Janeiro are gratefully acknowledged for financial support.

with 


\section{References}

[1] L. Baulieu and B. Grossman, Phys. Lett. B214, 223 (1988).

[2] D. Birmingham, M. Blau, M. Rakowski and G. Thompson, Phys. Rep. 209, 129 (1991).

[3] F. Delduc, F. Gieres and S.P. Sorella, Phys. Lett. B225, 367 (1989); F. Delduc, C. Lucchesi, O. Piguet and S.P. Sorella, Nucl. Phys. B346, 313 (1990).

[4] E. Guadagnini, N. Maggiore ans S.P. Sorella, Phys. Lett. B255, 65 (1991); N. Maggiore and S.P. Sorella, Int. Journ. Mod. Phys. A8, 929 (1993); C. Lucchesi, O. Piguet and S.P. Sorella, Nucl. Phys. B395, 325 (1993).

[5] M. Werneck de Oliveira, M. Schweda and S.P. Sorella, Phys. Lett. B315, 93 (1993); L. Tataru and I.V. Vancea, Int. Journ. Mod. Phys. 11, 375 (1996); A. Boresch, M. Schweda and S.P. Sorella, Phys. Lett. B328, 36 (1994).

[6] M. Carvalho, L.C. Queiroz Vilar and S.P. Sorella, Int. Journ. Mod. Phys. A, Vol. 10, 27, 3877 (1995).

[7] E. Witten, Comm. Math. Phys. 117, 353 (1988); D.Birmingham and M. Rakowski, Phys. Lett. B272, 217 (1991); D.Birmingham and M. Rakowski, Phys. Lett. B273, 74 (1991); M. Mariño, The Geometry of Supersymmetric Gauge Theories in Four Dimension, US-Ft-2/97, hep-th/9701128; F. Fucito, A. Tanzini, L.C.Q. Vilar, O.S. Ventura, C.A.G.Sasaki and S.P. Sorella, Algebraic Renormalization: Perturbative twisted consideration on topological Yang-Mills theory and on $N=2$ supersymmetric gauge theories, CBPFNF-043/97, hep-th/9707209.

[8] O. Piguet and S.P. Sorella, "Algebraic Renormalization ", Monographs Series, Vol. m28, Springer-Verlag, Berlin 1995.

[9] H. Nishino and S.J. Gates Jr., Int. Journ. of Mod. Phys. A, Vol.8, No. 19, 3371 (1993).

[10] S. Ouvry, R. Stora and P. Van Baal, Phys. Lett. B220, 159 (1989); J. Kalkman, Comm. Math. Phys. 153. 447 (1993); R. Stora, "Equivariant Cohomology and Topological Theories", in BRS Symmetry, M. Abe, N. Nakanishi, Iojima eds., Universal Academy Press, Tokyo, Japan, 1996;

R. Stora, F. Thuillier and J.C. Wallet, "Algebraic Structure of Cohomological Field Theory Models and Equivariant Cohomology", lectures given at the First Caribbean Spring School of Mathematics and Theoretical Physics, R. Coquereaux, M. Dubois-Violette and P. Flad Eds., World Scientific Publ., 1995; R. Stora, "Exercises in Equivariant Cohomology", ENSLAPPA-619/96, hep-th/9611114;

R. Stora, "De la fixation de jauge consideree comme un des beaux arts et de la symetrie de Slavnov qui s 'ensuit ", ENSLAPP-A-620/96, hep-th/9611115;

R. Stora, "Exercises in Equivariant Cohomology and Topological Theories", hep-th/9611116. 